\title{
Factores protectores de las familias para prevenir el consumo de drogas en un municipio de Colombia
}

\author{
Núbia Medina Arias ${ }^{1}$ \\ Maria das Graças Carvalho Ferriani²
}

\begin{abstract}
El objetivo de este estudio fue analizar los factores protectores de prevención del consumo de drogas, presentes en familias con niños que asisten a Hogares Comunitarios de Bienestar Familiar en un municipio colombiano. El estudio es de tipo descriptivo y cuantitativo transversal; la muestra estuvo constituida por 256 familias, seleccionadas por muestreo aleatorio simple. El instrumento para la recolección de datos fue un cuestionario aplicado durante los meses de marzo y abril de 2007, de forma autoaplicada. Se encontraron factores protectores como demostraciones de afecto con los hijos, jugar y hablar con ellos sobre lo que les gusta, comunicación fácil, toma de decisiones en pareja, adecuada flexibilidad durante la educación familiar, y existencia de normas. Por otro lado existen factores de riesgo como el consumo de drogas lícitas (cigarrillo y alcohol) y bajo porcentaje de drogas ilícitas. Un alto porcentaje de familias considera que los padres, principalmente, deben tomar medidas de prevención en los primeros años de vida del niño. Los factores protectores encontrados deben ser reforzados, debido a que no son muy fuertes; también, se deben controlar los factores de riesgo encontrados para convertirlos en factores protectores.
\end{abstract}

Descriptores: Factores de Riesgo; Drogas Ilícitas; Família.

\footnotetext{
${ }_{1}^{1}$ Profesor Asociado, Facultad de Enfermería, Universidad Nacional de Colômbia. E-mail: nsmedinaa@unal.edu.co.

2 Enfermera, Doctor en Enfermería, Profesor Titular, Escola de Enfermagem de Ribeirão Preto, Universidade de São Paulo, Centro Colaborador de la OMS para el Desarrollo de la Investigación en Enfermería, Brasil. E-mail: caroline@eerp.usp.br.
}

Correspondencia:

Maria das Graças Carvalho Ferriani

Universidade de São Paulo. Escola de Enfermagem de Ribeirão Preto

Av. Bandeirantes, 3900

Bairro Monte Alegre

CEP: 14040-902 Ribeirão Preto, SP, Brasil

E-mail: caroline@eerp.usp.br 


\title{
Fatores protetores das famílias para prevenir o consumo de drogas em um município da Colômbia
}

O objetivo deste estudo foi analisar os fatores protetores, visando prevenir o consumo de drogas, presente nas famílias de crianças atendidas nos Lares Comunitários do Bem-estar Familiar, em um município colombiano. Esta pesquisa é de cunho quantitativo descritivo transversal. A amostra foi constituída por 256 famílias selecionadas por amostra aleatória simples. $\mathrm{O}$ instrumento de coleta de dados foi um questionário usado no período de março a abril de 2007, de forma autoaplicavel. Foram encontrados fatores protetores como demonstrações de afeto com os filhos, brincar e falar com eles sobre o que eles gostam, a fácil comunicação, tomada de decisões pelo casal, adequada flexibilidade na criação e existência de normas. Por outro lado, existem fatores de risco, também, como o consumo de drogas lícitas como o cigarro e álcool, e, em baixa porcentagem, de drogas ilícitas. Uma alta porcentagem das famílias acha que os pais, principalmente, devem realizar a prevenção, nos primeiros anos de vida dos filhos. Os fatores protetores encontrados necessitam ser reforçados já que não são muito fortes, e devem ser monitorados os fatores de risco para serem transformados em fatores protetores.

Descritores: Fatores de Risco; Drogas Ilícitas; Família.

\section{Protective Factors for Preventing the Use of Drugs in the Families of a Colombia Locality}

\begin{abstract}
The aim of this study was to analyze the protective factors that prevent drug use in the families of children who attend Community Homes of Family Well-being in a small Colombian locality. This was a quantitative, descriptive, transversal study, with 256 families constituting the sample. Data were collected through a self-applied questionnaire throughout March and April 2007. Protective factors found included demonstrations of affection toward the children, playing with them and talking to them about things they like, open communication, decision making as a couple, flexibility of the nursing process, and establishment of rules. However, some risk factors were also found, such as consumption of legal drugs such as cigarettes and alcohol, and in a low percentage, consumption of illicit drugs. A high percentage of families consider that drug use must be prevented in the first years of life, by the parents. The protective factors found require reinforcement as they are not very strong, and the risk factors must be controlled to turn them into protective factors.
\end{abstract}

Descriptors: Risk Factors; Street Drugs; Family.

\section{Introducción}

Colombia es un país altamente afectado por la problemática de las drogas. Los cambios económicos, políticos y sociales ocurridos en los últimos años, en el país, han cambiado la realidad frente a las drogas; se ha pasado de ser un país productor y de trafico a un país consumidor, con tendencia al aumento en el consumo, a la disminución de la edad de inicio y a la utilización de nuevas sustancias, como se deduce de los informes presentados

por la Presidencia de la República al Mecanismo de Evaluación Multilateral"(1) en el año 2002 o el presentado en la Cumbre mundial de la infancia, en 2001.

Estos cambios han generado a su vez cambios sociales, estructurales e ideológicos, que repercuten en las familias, creando a veces condiciones de riesgo frente al consumo y otras veces manteniendo comportamientos que se constituyen en factores protectores. 
"La familia puede favorecer o desestimular el consumo de drogas. Una familia permisiva y sin normas claras puede convertirse en un factor que propicia el consumo. La familia que está cerca y cumple una función de regular a sus miembros en el cumplimiento de normas, aparece como factor protector para el consumo de Sustancias Psicoactivas (SPA) ${ }^{(2)}$.

La literatura sobre factores protectores en el consumo de drogas considera los hechos de los padres vivir juntos y cuidar de los hijos, la comunicación fácil, la buena percepción del padre y la buena integración en el hogar, como factores protectores(3).

El desarrollo de un adecuado funcionamiento familiar protege también a los jóvenes contra la influencia de otros factores de riesgo ajenos a la familia(4).

Entre los factores de protección personales y sociales se destacan, a nivel personal: mayor tendencia al acercamiento y a la empatía, mayores niveles de autoestima e incentivación al logro, sentimientos de autosuficiencia, autonomía e independencia, y actividades dirigidas a la resolución de problemas. A nivel social se destacan: ambiente cálido, comunicación abierta en el interior de la familia, estructura familiar sin disfunciones importantes, padres estimuladores y mayor apoyo social (emocional, material e informativo)(5).

Reconocido el papel vital que desempeña la familia en la niñez, periodo de la vida en que se sientan las bases de la personalidad y se generan muchos de los hábitos, se debe enfocar en ella la atención como el primer ámbito de prevención e intervención.

Teniendo en cuenta todo lo anterior, la niñez es considerada la época más propicia para iniciar las actividades de prevención del consumo de sustancias psicoactivas, y ésta debe hacerse, preferencialmente en el hogar. La responsabilidad primaria de prevenir está en las personas más cercanas al niño y al joven, que son sus padres y sus maestros.

La situación anteriormente descrita muestra la necesidad de generar programas de prevención frente al consumo, que con un enfoque holístico y de desarrollo del ser humano, eviten el aumento y abuso del consumo de drogas, legales e ilegales, y que mejor lugar que el núcleo familiar para desarrollar esta labor. Los profesionales del área de la salud, tienen la responsabilidad de promover y facilitar el alcance de los objetivos de desarrollo del ser humano, especialmente aquellos encargados del cuidado y atención a los niños, independientemente de su posición con respecto al tema del uso de sustancias psicoactivas $^{(6)}$.

Durante mi experiencia de trabajo como enfermera con las familias de niños preescolares, encontré que los padres muchas veces "recitan" prácticas saludables pero, no se evidencia en la realidad la aplicación de estos conocimientos.

Además, por ser la edad de sus hijos tan corta, los padres no expresan la necesidad de hablar sobre drogas, lo que si ocurre con los padres de adolescentes, lo cual puede atribuirse al desconocimiento sobre la necesidad de prevención en edades tempranas; pensar que en su entorno familiar no existen riesgos para el consumo o desconocer que ellos, como padres, ejercen un rol vital en la formación de sus hijos, son aspectos que deben ser reconocidos, ya que ellos pueden constituirse en factores protectores frente al consumo.

Informes verbales obtenidos en la Secretaría de Educación, de Salud y Secretaría Social del municipio de Funza, confirman el problema del consumo de drogas, pero reconocen la no existencia de estudios sobre éste en el municipio, desconociéndose así las prevalencias de consumo, tipos de drogas utilizadas y otros aspectos relacionados con el tema.

\section{Objetivo}

Identificar y analizar los factores protectores presentes para prevenir el consumo de drogas en familias con los niños que asisten a Hogares Comunitarios de Bienestar Familiar, en el municipio de Funza, Colombia.

\section{Método}

El estudio es descriptivo, con abordaje cuantitativo transversal. El escenario fue el municipio de Funza, cuya población se estima, según censo del año 2005 del Departamento Administrativo Nacional de Estadística(7) en 59.453 habitantes. Se calcula que posee un total de 15,792 hogares.

La Secretaría local de Salud y Medio Ambiente(8) del Municipio de Funza en el año 2000, identificó riesgos ambientales, biológicos y sociales. Entre estos últimos se encuentran la proliferación de pandillas juveniles en barrios, el desempleo y la falta de actividades recreativas y culturales que permitan mayor utilización del tiempo libre. Entre los eventos que prioritariamente figuran en este perfil epidemiológico se encuentra el alcoholismo $y$ 
la drogadicción, los cuales tienen como factor de riesgo el formar parte de una pandilla y la falta de actividades de promoción y prevención.

Funza cuenta con 37 Hogares Comunitarios de Bienestar Familiar para apoyar a las familias de menores recursos socioeconómicos en el cuidado de sus hijos.

La población estuvo constituida por (523) el $100 \%$ de las familias de los niños preescolares que se encontraban registrados y asistían a ellos.

La muestra la constituyen 256 (48,9\%) familias; número que estaba por encima del tamaño mínimo representativo, al hacer la simulación de los coeficientes de variación estimados en la prueba piloto. La muestra se seleccionó por Muestreo Aleatorio Simple (MAS) con un listado actualizado de todas las familias, y con auxilio del programa computacional que seleccionó los números de forma aleatoria. Se establecieron como criterios de inclusión:

1. Ser familias de niños matriculados y participando de los Hogares Comunitarios de Bienestar Familiar del Municipio.

2. El familiar informante debe ser mayor de edad y con capacidad mental para comprender y responder.

El cuestionario de formato autoaplicado, fue elaborado por la investigadora con prueba piloto y panel de expertos, basada en el instrumento "Cómo es tu familia", de la Organización Panamericana de la Salud, OPS ${ }^{(9)}$. La prueba piloto identifico la comprensión de 19 preguntas y la necesidad de hacer ajustes en tres de ellas, lo cual se hizo hasta lograr su absoluta comprensión. Se utilizó el formato autoaplicado por ser un método eficiente para estimar consumo de drogas porque garantiza el anonimato.

El cuestionario, de 22 preguntas cerradas, contiene ítems sobre descripción sociodemográfica de la familia, consumo de drogas por parte de miembros de la familia, conceptos de los padres sobre prevención del consumo de drogas, afecto y comunicación en la familia, y educación familiar de los hijos.

Los datos se recolectaron durante los meses de Marzo a Abril de 2007. Se solicitaron y obtuvieron los permisos correspondientes. En las reuniones mensuales de cada Hogar Comunitario, se dio a las familias información amplia sobre el estudio y se obtuvo su participación voluntaria, se explicó el manejo confidencial de la información, y luego se procedió a autoaplicar el cuestionario a aquellas familias que voluntariamente desearon hacerlo. Para preservar la privacidad lo hicieron en el mismo Hogar Comunitario, cada familia separadamente; luego se recogieron los cuestionarios y el término de Consentimiento Informado, esto realizado de manera a preservar el anonimato de los informantes.

El Término de Consentimiento Informado tuvo en cuenta los principios de confidencialidad y autonomía contemplados en la Resolución 8430 de 1993, que establece las Normas Científicas, Técnicas y Administrativas para la investigación en Salud, en Colombia. Una vez aplicados los cuestionarios se procedió a su análisis con los paquetes estadísticos SPSS versión 10.0 y SPAD versión 4.5.

\section{Resultados}

\section{Características sociodemográficas de las familias}

El $83 \%$ de la población estudiada (212 familias) llevaba viviendo más de 3 años en Funza. Solo un 5\% (13 familias) Ilevaba menos de un año viviendo allí y procedían de ciudades cercanas.

De los 256 informantes (197), el 77\% corresponde a madres; algunas de las cuales asumen el rol de jefe cabeza de hogar; $2,3 \%$ (6) fueron abuelas y un $17,2 \%$ (44) padres hombres. En 10 familias existía un solo progenitor.

Con relación a la escolaridad de los padres y madres, el 2\%, (4) no tenían ningún grado de educación. El mayor porcentaje de escolaridad correspondió al nivel de bachillerato completo, para los padres 34\% (15) y $41 \%$ (81) para las madres. El nivel educativo de las madres es ligeramente superior al de los padres. El $8 \%$ de los padres y madres (20) tienen nivel tecnológico.

El 57\% (25) de los padres tenían edad superior a 28 años y solamente $44 \%$ de las madres tenían edad superior a 28 años. No se identificó padres o madres menores de 18 años de edad. 31\% de los padres y madres (79) se encontraban en edades entre 19 y 22 años.

\section{Consumo de drogas en las familias}

Con relación al consumo de drogas lícitas, en 50\% de las familias se consume cigarrillo y en $26 \%$ de éstas se hace diariamente (Figura 1 ). 


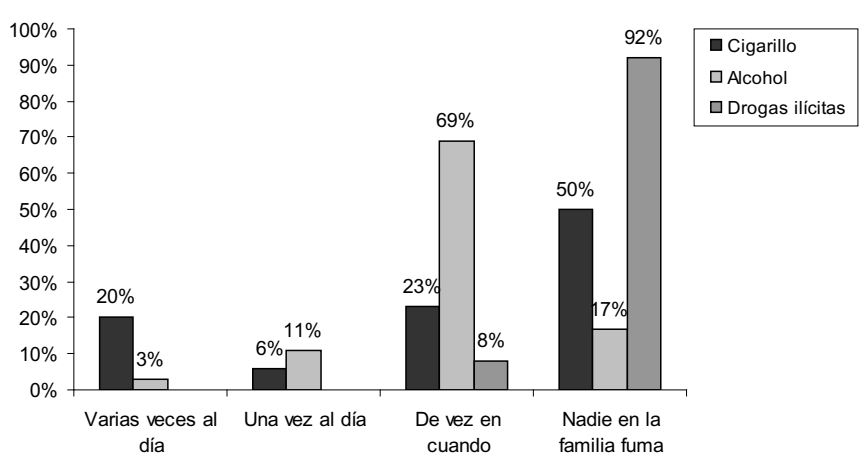

Figura 1 - Consumo de drogas lícitas e ilícitas en familias. Funza, Colombia, 2007.(n 526)

A pesar de que en un $50 \%$ de las familias (128) hay consumo, 59\% (151) expresa desacuerdo con éste, $15 \%$ (38) expresa acuerdo y el 26\% (67) indiferencia. Sumadas las familias para las cuales es indiferente $y$ aceptado el consumo de cigarrillo, se aprecia una cifra alta, (41\%), de familias (105) en las cuales podría consumirse.

En el $83 \%$ de las familias se bebe, en $14 \%$ de ellas semanalmente y solo en $17 \%$ de las familias (43) no hay consumo de alcohol. Frente a la aceptación, 23\% de las familias (60) lo aceptan, para el 19\% (48) es indiferente y el 58\% (148) está en desacuerdo.

Con relación a las drogas ilícitas, en el $92 \%$ de las familias (235) no existe consumo, a pesar de que solamente 66\% (170) expresa estar en desacuerdo con éste consumo; las familias indiferentes fueron el $31 \%$ (79) y las que aceptan un 3\% (7). La aceptación del consumo en la forma - de vez en cuando - solo se encontró en $8 \%$ de las familias (21), lo contrasta con las cifras de aceptación o indiferencia (86).

\section{Opinión de las familias sobre la prevención del consumo}

El 90\% de las familias considera que la Prevención deben hacerla los padres, seguido de otros familiares, cuando se trata de un menor de edad $69 \%$. El $1 \%$, considera que nunca debe hacerse.

Un buen porcentaje cree que debe hacerse prevención con el diálogo $89 \%$ y con el ejemplo $72 \%$. Aparecen también las conferencias y talleres.

\section{Factores protectores}

Afecto. Jugar y hablar con ellos sobre lo que les gusta; a pesar de no hacerse a diario, está presente en (130) $51 \%$ de las familias.
Con relación a las demostraciones de afecto utilizadas generalmente en las familias, se encontró "el compartir tiempo" como la más utilizada, seguida de "caricias", "regalos" y "otras". Se encontró 2\% de familias que expresaron no tener ninguna demostración de afecto.

Comunicación. Es siempre fácil para 34\% de estas familias y solamente fácil "a veces" en $59 \%$ de ellas. $7 \%$ de las familias (18) consideran siempre difícil la comunicación. El $31 \%$ (79) comparten una sola comida.

La solución de conflictos en $70 \%$ de las familias (180) es a través del diálogo. Otras formas de resolución identificadas fueron apartarse 8\% (21), ofensas verbales $9 \%$ (23), resolución viene con el tiempo $9 \%$ (23), 3\% (7) con ofensas físicas y un $1 \%(2)$ lo resuelve de otras formas, entre las que aparecen la oración.

Con relación a quién toma generalmente las decisiones en la familia se encontró que en un $56 \%$ de las familias (143) se toman en pareja, 32\% (82) uno solo de los padres y solo en un $12 \%$ (31) lo hacen los padres con participación de los hijos.

Cuando los hijos están en dificultades expresan que, acuden $82 \%$ a la madre y $62 \%$ al padre.

Normas. En el $56 \%$ de las familias (143) existen normas conocidas y que se cumplen, no así en el $21 \%$ de las familias (54) donde existen pero no se cumplen y en $23 \%$ (59) donde no son claras y por eso no se cumplen.

Educación familiar. Cómo se reprenden los hijos generalmente en la familia; 49\% (124) lo hacen explicándoles su error, 29\% (72) prohibiéndoles algo que les gusta, 21\% (54) con una palmada o "correazo" y en $2 \%$ de las familias (6) no les dicen nada. 


\section{Discusión}

\section{Características sociodemográficas}

El hecho de llevar viviendo el $83 \%$ de la población más de 3 años en Funza, se constituye en factor protector, por cuanto esto les permite un buen conocimiento del sector.

El ser la mayoría de informantes las madres lleva a pensar que fue una buena fuente.

El hallazgo de algunas familias (10) con presencia de un solo progenitor, por separación o divorcio, vale la pena que se considere, ya que esto se constituye en un factor de riesgo según varios autores; "vivir fuera del hogar, alienación de la familia, hogares rotos", entre otros $^{(10)}$. En una revisión de trabajos relacionados con la familia del adicto se encontró una alta incidencia de familias de origen quebrantadas ${ }^{(5)}$.

En la investigación sobre Factores protectores y de riesgo en el consumo, en el caso de Actitud hacia la familia, se encontró como factores protectores el hecho que los padres vivan juntos, cuiden de los hijos, y la buena integración en el hogar(3).

El bajo nivel de escolaridad de los padres, en este caso $2 \%$ sin ningún grado de educación y solo $34 \%$ de los padres bachilleres, es también señalado como factor de riesgo por algunos investigadores ${ }^{(11)}$ quienes encontraron que existe un perfil de vulnerabilidad predictivo del consumo de drogas en hijos adolescentes, cuyos factores más importantes fueron: la baja cohesión familiar, las dificultades para tomar decisiones y solucionar sus problemas con la participación democrática de todos sus miembros, dificultades en la comunicación entre padres e hijos, y bajo nivel de instrucción de los padres.

Con relación a la edad de los padres, el $31 \%$ de ellos (79) se encontraba en edades entre 19 y 22 años y no hubo menores de 18 años, lo que fue identificado también como factor protector frente al consumo de drogas. Pues se señala que en la etapa experimental, la familia presenta indicadores de alto riesgo; como modelos educativos inadecuados, alienación sociocultural, trastornos de la comunicación, inmadurez de las figuras significativas, simbiosis, desafecto, liberalismo excesivo, ausencia de medidas de control social y de alerta contra el uso de drogas(12).

\section{Consumo de drogas en las familias}

El consumo de cigarrillo en $50 \%$ de las familias evidencia la presencia alta de consumo si tenemos en cuenta que hay consumo diario, lo cual de acuerdo con la literatura es un factor de riesgo que induce o propicia el consumo ${ }^{(13)}$

La aceptación e indiferencia expresada hacia el consumo de cigarrillo podría llevar a él, sin ser censurado o tener alguna objeción por parte de otros miembros de estas familias, con el consiguiente riesgo para el consumo en los menores.

Como reporta el III Estudio Nacional de Salud Bucal(14) "quienes continúan fumando como adultos, es porque quedaron enganchados en esta adicción y les es imposible abandonarla" motivo por lo cual este resultado ameritaría explorar más con estas familias para conocer a fondo el por qué de ese consumo; o como lo evidenció el estudio

"Creencia en tabaquistas"(15) donde el 60\% de los sujetos manifestaron haber intentado dejar el consumo de cigarrillo, mostrando su deseo contradictorio con su actuar.

El consumo de bebidas alcohólicas en $83 \%$ de las familias contrasta con el desacuerdo expresado en $58 \%$ de éstas, y la aceptación e indiferencia hacia su consumo, podría llevar igual que con el cigarrillo, a consumirlas sin ser censurado o tener alguna objeción por parte de otros miembros de la familia.

Que $92 \%$ de las familias no consuma drogas ilícitas se evidencia claramente como factor protector de los menores para evitar el consumo de este tipo de sustancias. La literatura señala como factores de riesgo la aprobación del uso del alcohol o de la droga, el abuso del tabaco, alcohol o de otras drogas por parte de los padres, y la historia de alcoholismo en la familia(5). Se enfatiza que una actitud permisiva o el mismo consumo de drogas en los progenitores pueden promover un aprendizaje por imitación de modelo y facilitar la incorporación del menor a grupos de consumidores(16). Otras investigaciones muestran también esta tendencia o asociación ${ }^{(5)}$.

\section{Opinión de las familias sobre la prevención del consumo}

Coherente con considerar que los padres y la familia tienen la responsabilidad de hacer prevención, un buen porcentaje cree que debe hacerse con el diálogo y con el ejemplo. Estos resultados confirman la necesidad de iniciar la prevención del consumo de drogas en edades tempranas, directamente con las familias.

Los profesionales del área de la salud tienen la responsabilidad de promover y facilitar el alcance de los objetivos de desarrollo del ser humano, especialmente 
aquellos encargados del cuidado y atención a los niños, independientemente de su posición con respecto al tema del uso de sustancias psicoactivas ${ }^{(6)}$. $Y$ son entre ellos, las enfermeras, quienes en mayor número, están más tiempo en contacto con las personas y las comunidades.

\section{Factores protectores}

Afecto. Las demostraciones de afecto como jugar y hablar se identifican como factores protectores que, sin embargo, requieren ser reforzados y comprendidos por los padres para que se realicen diariamente en todas las familias, pues $^{(5)}$, por otro lado, la falta de relaciones fuertes y de cariño es un factor de riesgo en la familia frente al consumo de drogas, "la falta de lazos familiares adecuados, propicia un perfil de adolescente incapaz de establecer relaciones saludables" lo cual lo hace más vulnerable frente al consumo(11).

Además, en el mundo actual, los padres se ven obligados a trabajar fuera del hogar, lo cual ocasiona una reducción en el tiempo y atención prestados a los hijos y hace necesario que otras instituciones tengan que asumir nuevas responsabilidades que tradicionalmente no les correspondía(4).

\section{Comunicación}

Que la comunicación no sea siempre fácil en estas familias, a pesar de la corta edad de sus hijos, se constituye en factor de riesgo frente al consumo. Por el contrario, entre los factores de protección personales y sociales para el consumo mencionados en la literatura están la existencia de madres o sustitutas que dan apoyo, la comunicación abierta en el interior de la familia, y el mayor apoyo social (emocional, material, informativo, formación de valores) $)^{(5)}$.

La resolución de conflictos a través del diálogo se encontró solamente en $70 \%$ de las familias y la toma de decisiones por solo uno de los padres en 32\%, se constituyen también en factores de riesgo. Existe un perfil de vulnerabilidad predictivo del consumo de drogas en hijos adolescentes, cuyos factores más importantes son la baja cohesión familiar y las dificultades para tomar decisiones y solucionar sus problemas con la participación democrática de todos sus miembros. Un estudio comparativo de familias normales y familias con un miembro adicto, encontró que la familia con un usuario de drogas mantenía una gran rigidez en la toma de decisiones ${ }^{(5)}$.
Algunos factores de riesgo tales como la falta de comunicación, ocasionados principalmente por la dificultad para escuchar, o responder adecuadamente por parte de los padres, se reconocen como factor de riesgo(17). La buena relación con los hermanos, el llevarse bien en casa, la comunicación fácil y la buena integración en el hogar, son factores protectores ${ }^{(3)}$.

Al concluir que los factores vinculados a la relación familiar juegan un rol decisivo en el comportamiento adictivo(17), se dice que, la falta de comprensión y comunicación es un factor de riesgo para el uso de drogas ilegales. En sentido opuesto, el encontrar un medio familiar en el que sucede la comunicación y el intercambio de opiniones, aparece como factor de protección al uso de drogas.

\section{Normas}

El hecho de que en un $44 \%$ de las familias no existan normas o no ellas no sean claras, es un factor también de riesgo pues "una familia permisiva y sin normas claras puede convertirse en un factor que propicie el consumo. La familia que está cerca y cumple una función de regular a sus miembros en el cumplimiento de normas de vida, aparece como factor protector para el consumo de SPA(2)

Otro factor de riesgo señalado es la dificultad para fijar límites: La permisividad acarrea dificultades para interiorizar normas debido a que la ausencia de ellas en el entorno familiar impedirá, la generalización a otros medios. La rigidez genera falta de flexibilidad en el cumplimiento de las normas o una tendencia a la trasgresión de las mismas. La exigencia desmedida favorece la falta de motivación y añade dificultades para aceptar los fracasos(17).

La literatura establece también, que el liberalismo excesivo y la ausencia de medidas de control social y de alerta contra el uso de drogas, son indicadores de alto riesgo como modelos educativos inadecuados(12).

Educación familiar. Reprender a los hijos con palmadas o correazos (21\%) o "no diciéndoles nada" fue otro factor de riesgo identificado ya que se señalan como factores de riesgo para el consumo de drogas: vivir fuera del hogar, hogares rotos, control parental relajado(18).

Una investigación en la que se analizaron 96 familias de drogadictos mostró también que en aquéllas familias en las que había un alto grado de control parental, una elevada ambición de logro con su consiguiente alto nivel 
de expectativas, donde había actividades compartidas, existía una probabilidad más baja de consumo de drogas. Si cualquiera de estas actitudes es exagerada, entonces lo inverso puede ser realidad. Actitudes parentales de sobreprotección, pero de excesivas e inefectivas demandas pueden conducir o perpetuar el abuso de $\operatorname{drogas}^{(5)}$.

\section{Conclusiones}

Un primer factor protector identificado, lo constituye la permanencia de las familias en Funza, por más de 3 años, al igual que la edad de los padres; adultos jóvenes.

Se encontraron algunas familias monoparentales; lo cual de acuerdo a algunas investigaciones ${ }^{(3)}$ se constituye en factor de riesgo.

Otro factor de riesgo identificado fue el consumo de drogas lícitas como cigarrillo y alcohol, siendo el consumo de bebidas alcohólicas mayor que el de cigarrillo; también, el consumo de drogas ilícitas, encontrado en bajo porcentaje de las familias estudiadas.

Lo anterior plantea la necesidad de realizar futuras investigaciones para determinar la prevalencia en el consumo y el desacuerdo frente a éste y así lograr correspondencia entre el pensar y el actuar. Igualmente se requiere controlar los factores de riesgo encontrados para convertirlos en factores protectores.

Un alto porcentaje de familias 69\% (176) considera que la prevención debe hacerse en los primeros años de vida de la persona, lo que es coherente con lo evidenciado por algunos autores y llama la atención que hay familias, aunque son pocas, que parecen no tener conocimiento sobre el tema.

La familia y los maestros son considerados por las familias como los principales responsables por hacer la prevención del consumo y muestra el estudio que el personal de salud no es visto por ellos tan significativo para esta tarea.

Se requiere reforzar factores protectores presentes en estas familias como las demostraciones de afecto con los hijos, jugar y hablar con ellos sobre lo que les gusta, la comunicación, la toma de decisiones en pareja, la adecuada flexibilidad en la educación familiar, y la existencia de normas.

Se recomienda mayor participación de enfermería en el abordaje del tema de consumo de sustancias psicoactivas, por ser un profesional que posee herramientas conceptuales y metodológicas para hacerlo y desarrollar investigaciones que enriquezca el conocimiento.

Con base en los resultados del estudio se espera por parte de las entidades municipales responsables de los Hogares y con asesoría técnica de la investigadora, fortalecer en estas familias los factores protectores identificados e iniciar el control de factores de riesgo encontrados.

El estudio permite contribuir desde el abordaje integral del cuidado de enfermería a la confirmación de la importancia e influencia de la familia para el ser humano; también permite identificar la existencia o no en ellas de factores protectores frente al consumo de drogas, lo que permitiría generar programas de prevención enfocados en el ámbito familiar, ya que como muestran estudios recientes ${ }^{(18-19)}$, los jóvenes reconocen el papel que juega la familia en este tema, sea en el ámbito de formación personal o profesional.

\section{Agradecimientos}

Agradecemos a la Comisión Interamericana para el Control del Abuso de Drogas/CICAD de la Secretaria de Seguridad Multidimensional/SSM de la Organización de los Estados Americanos/OEA, la Secretaria Nacional de Políticas sobre Drogas/SENAD do Gabinete de Seguridad Institucional/Brasil, la Escuela de Enfermería de Ribeirao Preto de la Universidad de Sao Paulo y Centro Colaborador de la Organización Mundial de la Salud para el Desarrollo de la Investigación en Enfermería, la población representada en los estudios de investigación, bien como a las autoridades de las universidades representadas por los participantes del Programa En-Line de Especialización en Investigación sobre el Fenómeno de las Drogas PREINVEST, periodos 2005, 2006, 2007 y 2008.

\section{Referencias}

1. Mecanismo de Evaluación Multilateral, MEM. 2001-2002. Colombia.Evaluación del Progreso de Control de Drogas. Washington, D.C: Organización de los Estados Americanos-OEA. Comisión Interamericana para el Control del Abuso de DrogasCICAD; 2002.
2. Téllez, J, Cote M, Savogal F, Martínez E, Cruz U. Identificación de factores protectores en el uso de sustancias psicoactivas en estudiantes universitarios. Rev Fac Med. (Bogotá) ene.-mar. 2003; 51(1):15-24.

3. Perotto V. El riesgo de ser joven. Investigación: Factores protectores y de riesgo en el consumo de drogas. La Paz: CESE; 1994. 
4. Fragüela JAG. El papel de la familia en el consumo recreativo de drogas.[s.d.]. Santiago de Compostela: Universidad Santiago de Compostela, España. Facultad de psicología. [Acceso Septiembre 10, 2006]. Disponible en: http:/www.ceapa.es 5. Ugarte RM. La familia como factor de riesgo, protección y resiliencia en la prevención del abuso de drogas en adolescentes. In: Vargas AZM, editor. Factores de riesgo y protección en el consumo de drogas en la juventud. Perú: CEDRO, 1999.

6. Barrios AM, Rueda PE. El pediatra como agente de protección en el uso de psicoactivos. En: Memorias del 2do Encuentro de Salud integral y sustancias psicoactivas. Bogotá (CO): Universidad Nacional de Colombia. Dirección de Bienestar; 2002.

7. Departamento Nacional de Estadística. DANE (CO). Censo 2005. [Acceso en: Septiembre 10, 2006]. Disponible en: http:// www.dane.gov.co/censo/

8. Secretaria de Salud y Medio Ambiente (Funza-CO). Perfil epidemiológico. Funza, Cundinamarca: Alcadia de Funza; 2000. 9. Organización Panamericana de la Salud. OPS. Organización Mundial de la Slud, OMS. Familia y adolescencia: Indicadores de salud: Manual de aplicación de instrumentos. Washington, D.C.: OPS/ OMS., Diciembre 1996.

10. Organización Mundial de la Salud. OMS Reporte Técnico No $731 ; 1986$.

11. Uugarte Díaz RM. La familia como factores de riesgo, protección y resiliencia en la prevención del abuso de drogas en adolecentes [Internet]. Disponible en: http://www.cedro.org.pe/ebooks/ friesgo_cap5_p130_169.pdf
12. Martín N. Estudio fenomenológico descriptivo de 120 pacientes dependientes principalmente a pasta básica de cocaína. [Tesis Doctoral]. Universidad Nacional Mayor de San Marcos. Lima. Peru; 1991.

13. Rueda AR. Qué son las drogas. Unidad coordinadora de prevención integral. Dirección Nacional de estupefacientes. Unidad de coordinación Nacional. Bogotá, Colombia; 1992.

14. Ministerio de Salud. Colombia. III Estudio Nacional de Salud Bucal; 1999.

15. Gonzalez RC. Creencias en fumadores pertenecientes a un programa de salud cardiovascular. Rev Latino-Am. Enfermagem. março-abril 2004;12(Número especial):412-9.

16. Sancho JLA. Familia, adolescentes y consumo de drogas. [Acceso en: 10 Septiembre 2006]. Disponible en: http://www. alcoholinformate.org.mx/portal_jovenes/home.cfm?familia

17. Rojas M. Factores de Riesgo y Protección. En: El abuso de drogas ilegales en adolescentes jóvenes de Lima Metropolitana. Lima: CEDRO; 1999.

18. Montoya de Abarca, Pillon SC. Percepción de las estudiantes de enfermería sobre los predictores del uso de drogas. Rev. Latino-Am. Enfermagem. 2008 julio-agosto; 16 (especial):607-13.

19. García de Jesús MC, Ferriani MGC. La escuela como "factor protector" para las drogas. Rev. Latino-Am. Enfermagem. 2008 maio-junho; 16(especial):590-4. 